\section{Review}

Correspondence

Timothy J. J. Inglis

tim.inglis@health.wa.gov.au

\title{
Principia ætiologica: taking causality beyond Koch's postulates
}

\author{
Timothy J. J. Inglis
}

Division of Microbiology and Infectious Diseases, PathWest Laboratory Medicine WA, OEII Medical Centre, and School of Biological and Chemical Sciences, University of Western Australia, Nedlands, WA 6909, Australia

\begin{abstract}
There is no single accepted method to establish a causal relationship between an infective agent and its corresponding infectious disease. Different biomedical disciplines use a patchwork of distinct but overlapping approaches. To a greater or lesser extent these are based on criteria known as the Koch-Henle postulates, or 'Koch's postulates' for short. Deficiencies in Koch's postulates were recognized by their principal author shortly after their formulation. Now, over a century later, a more rigorous method to test causality has still to be finalized. One contender is a method that uses molecular methods to establish a causal relationship ('molecular Koch's postulates'). Recognizing the wider range of contemporary approaches used to build an argument for a causal relationship, the use of a more inclusive approach to establish proof of causality is proposed. This method uses an argument built from a series of assertions. Assertion 1: congruence or reproducible correlation of a taxonomically defined life form with the clinico-pathological and epidemiological features of infection. Assertion 2: consistency of the demonstrable biological response in the subject to an encounter with the prospective infective agent. Assertion 3: progressive or cumulative dissonance as an explanation for pathophysiological processes at every known level of biological organization in the subject. Assertion 4: curtailment of that pathophysiological process on the deliberate introduction of a specified biomedical intervention. Evidence to implicate the candidate biological entity as an initiator of or primer for cumulative dissonance places it in a subcategory of micro-organisms to be known as 'priobes'. A priobe is the sufficient and necessary antecedent cause of a pathophysiological process evident as an infectious disease.
\end{abstract}

\section{Introduction}

Robert Koch and Jacob Henle are generally credited with the first method used to establish the aetiology of a specified infectious disease (Koch, 1884). The Koch-Henle postulates served the emerging science of microbiology well during its early years, and gave experimental consistency to the investigation of causal relationships. However, significant limits to the postulates were soon recognized and restricted their wider scientific application. In recent times, the original Koch-Henle concept of causation has been used by experimentalists with decreasing frequency. The expanding investigative repertoire of the life sciences dictates a need for a contemporary method for establishing the cause of infection. Falkow recognized the need for more rigorous genetic criteria for the determinants of a disease, and modelled his proposals for the molecular biology of pathogenesis on the Koch-Henle postulates (Falkow, 1988). While Falkow's molecular reiteration of the original postulates established a standard for molecular and cell biology developments in the clinical sciences, it did not anticipate a direct contribution to concepts of pathogenesis from the emerging fields of molecular ecology and microbial population genetics. The possibility that all levels of biological organization might have some bearing on the occurrence, course and outcome of infection was recognized by Colwell and named 'biocomplexity' (Colwell, 1999). Lederberg alluded to the evolutionary context of infection when he posed the question why do micro-organisms not cause disease more often (Lederberg, 1999). Salyers and Witt placed even greater emphasis on ecological determinants of bacterial infection (Salyers \& Witt, 2005). In their critique of the Koch-Henle postulates, they highlight the need to recognize that the effect of specific biomedical interventions might also provide proof of cause.

These considerations are undergirded by developments in scientific method and the philosophy of science. There is growing ethical objection to the use of laboratory animal models for incremental scientific gain. This places a restraint on the use of animal models for pathogenesis research or clinical diagnostic work. Molecular and cell biology methods cannot fully substitute for tissue pathology, and 
whole-organism outcome indices, such as failure to thrive, weight loss and death. There are also significant difficulties establishing an aetiological role for micro-organisms that cannot be cultivated under laboratory conditions. At a philosophical level, contemporary scientific method does not assume absolute objectivity in the experimentalist and places greater reliance on verification by complementary experimental methods. In the specific case of establishing cause, supportive evidence for sufficient cause (evidence that the proposed causal agent was capable of a causal role) is inadequate. Evidence for necessary cause must also be advanced. To establish necessary cause, there must be evidence that an outcome cannot occur without the proposed agent. In studies on the aetiology of infection, necessary cause alludes to Popper's concept of falsifiability, and to the recognition by Salyers and Witt of medical intervention as evidence for a causal relationship (Popper, 1963; Salyers \& Witt, 2005).

Central to our understanding of the origin or pathogenesis of infectious disease is the causal microbial agent or 'pathogen'. This term has acquired a range of overlapping and sometimes contradictory meanings as our knowledge of pathogenic processes has grown. Given these limitations, I will use 'priobe' as a new term to denote a specific, biological initiating or priming agent implicated as the sufficient and necessary antecedent cause of an infective process.

The principles by which confirmation of an aetiological role can be established for the inferred infective agent are laid out in the following paragraphs.

\section{Definition of terms \\ Priobes}

(i) A priobe is a specific, biological initiating or priming agent implicated as the sufficient and necessary antecedent cause of a pathological process.

(ii) All priobes are capable of initiating or priming a biological response in a dissimilar life form.

(iii) Not all micro-organisms are priobes.

(iv) Not all priobes are micro-organisms.

(v) Most but not all priobes are unicellular.

(vi) Most but not all priobes are capable of independent biological organization at molecular or higher level.

(vii) Failure of the responding life form to resolve negotiations with a priobe at any level of biological organization leads to accumulation of dissonant effects at that level and contributes to the pathological state.

(viii) Most but not all priobes must maintain an encounter with the responding life form to sustain cumulative dissonance.

\section{Cumulative dissonance}

(i) The progressive or accumulated series of changes initiated by a priobe at every level of biological organization in which the pathological state has been described.

(ii) Thus, an accumulation of unresolved negotiations in successive biological dimensions arising from encounters between distinct life forms determines the occurrence, course and outcome of pathology.

\section{Principles of aetiology}

(i) The method recognizes that the argument for a causal relationship will be assembled from a series of assertions about the interactive relationship between the proposed priobe and its corresponding biological partner. The sequence begins with investigation of conjecture, continues through an accretion of experimental evidence and concludes with demonstrable falsifiability of the core relationship through deliberate, controlled intervention.

(ii) The method aims to satisfy the requirements of the Koch-Henle postulates (Koch, 1884), Falkow's molecular corollary (Falkow, 1988), Colwell's recognition of scales of biocomplexity (Colwell, 1999), observations by Salyers and Witt on the admissibility of intervention (Salyers \& Witt, 2005) and Popper's insistence on falsifiability (Popper, 1963).

(iii) The method recognizes the wider biological context of an interactive process considered to be infection, and avoids attribution of causality from isolated experimental, pathological or time-placelimited epidemiological phenomena.

(iv) The method matches the level of certainty attached to a causal relationship with the number of assertions that can be made to support the argument.

(v) The method accepts that preliminary conclusions as to the causation of individual clinical episodes, public health incidents and the natural history of emerging infections are all provisional and subject to revision in the light of new observations.

\section{Argument \\ Assertion 1: congruence}

The consequences of an encounter with a taxonomically defined, distinct life form must be congruent with the clinical, pathological and epidemiological features of disease.

\section{Assertion 2: consistency}

The introduction of a taxonomically defined life form must initiate a consistent biological reaction without antecedent external cause.

\section{Assertion 3: cumulative dissonance}

The taxonomically defined life form must initiate a progressive series of changes at every level of biological organization in which the infection has been described. 


\section{Assertion 4: curtailment}

Restriction, alteration, destruction or removal of the candidate priobe at any level of biological organization must reduce the occurrence, course or outcome of the corresponding infection.

\section{Process}

The process of aetiological certainty is shown in Table 1.

\section{Practical application}

Before Koch's postulates there was no rigorous method to establish causality. However, the system he introduced in the postulates had methodological and epistemological limitations (Mazzarello, 2004). The immediate quandary Koch encountered was how his postulates could accommodate the recurrence of cholera epidemics in the absence of an asymptomatic carrier state or environmental reservoir. Until toxigenic Vibrio cholerae was discovered in the mucilaginous coating of blue-green algae, it was widely believed that cholera lacked an environmental reservoir (Islam et al., 1990; Alam et al., 2006). Further work on the ecology of $V$. cholerae, and its relationship to oceanic temperature fluctuations and climatic cycles, has added important detail to a biocomplexity model of cholera risk that spans the molecular and cellular scale at one end to human social behaviour and climatic change at the other (Lipp et al., 2002). Cholera has therefore become the test case for biocomplexity, over a century after Koch's original observations on the disease. The biocomplexity concept has been successfully employed as a model in reverse by beginning with a point-source outbreak, and pursuing the biocomplexity paradigm through individual patient management and cellular interactions in the environment, down to specific molecular markers for a disease of environmental exposure: melioidosis (Inglis et al., 1998, 2000, 2001; Merritt et al., 2006).

Another aspect of infectious disease that eluded Koch and his contemporaries was the basis of diseases that lacked a single precipitating microbe or microbe-derived toxin. The role of microbial consortia and polymicrobial complexes in specific infectious disease is now better understood through the contribution of biofilms, particularly in medical device-associated infections (Costerton et al., 2005). In the current model of causality, these biofilms can be considered as proto-tissues, entering the pathophysiological process at the tissue level of organization. These and other biofilm-related infections necessarily defy analysis in a one species/one organ model of infection, particularly when the immediate environment is dynamically changing, such as the respiratory support devices used for intensive care patients (Safdar et al., 2005).

It follows that the interface between infected subject and priobe requires analysis on both sides of the border. Microbe/innate immunity interactions do not always follow the simple course originally predicted. The consequences of an encounter with a microbe or its products can be a spectrum of evoked responses, which may in turn be modulated by the variable condition of the infected subject. A decade ago the variation in outcomes of severe sepsis was attributed to immunological dissonance: a breakdown in the balance between pro-inflammatory and suppressive effectors (Bone, 1996). This process was subsequently alluded to as an example of predictably complex biological phenomena in a dysfunctional upscaling of host response during severe infection (Gullo, 1999). The current synthesis argues that immunological dissonance is an important component of cumulative dissonance because it describes how the evoked host response can become dysfunctional and ultimately destructive. An example of damage incurred in human subjects as a result of cumulative dissonance is the cytokine storm proposed for some of the more severe pathophysiological aspects of epidemic influenza (Yokota et al., 2000). Although the earliest descriptions of the process describe cumulative dissonance in influenza, the other three criteria are still required to establish a causal relationship between a specific strain of influenza virus and specific disease events. Congruence, consistency and curtailment also provide yardsticks for more rigorous analysis of emerging infectious agents. They are and have been used extensively under different names by different biomedical disciplines, but need to be assembled in a single cohesive argument if truly new disease phenomena, such as severe acute respiratory syndrome, are to be better understood.

Possibly the greatest challenge to any novel approach to causal analysis of infectious disease is the group of proposed infective agents that have been linked with the transmissible spongiform encephalopathies (Aguzzi \& Heikenwalder, 2006). In some circles, viruses still provoke discussion about whether or not they constitute a

Table 1. The process of aetiological certainty

\begin{tabular}{|c|c|c|c|}
\hline $\begin{array}{l}\text { Aetiological } \\
\text { certainty }\end{array}$ & Assertion & Short form & Explanation \\
\hline Conjectural & 1 & Congruence & Congruence with clinico-pathological and epidemiological features \\
\hline Candidate & 3 & Cumulative dissonance & Progressive or cumulative dissonance at every known level of biological organization \\
\hline Confirmed & 4 & Curtailment & Curtailment of infective process on controlled introduction of biomedical intervention \\
\hline
\end{tabular}


legitimate life form. The proposed agents of spongiform encephalopathy are therefore right at the edge of contemporary understanding of microbial life. Yet they appear to behave as transmissible agents of disease. The prion hypothesis is thus a test of the present model for analysis of causation. From what is already known, there is a degree of congruence between the molecular biology, epidemiology and clinico-pathological features. This has reproducible consistency and elements of cumulative dissonance as the disease progresses during a period of latency from molecular and cellular damage to the onset of gross neurological signs. It could also be argued that the effects of the offal ban in the UK in response to the bovine spongiform encephalopathy outbreak represent evidence for curtailment (Stevenson et al., 2000). If the remaining details in the pathophysiology of Creuzfeldt-Jacob Disease continue to provide evidence for the proposed four-point argument for causality, the putative prion agent will deserve recognition as a provoked reaction initiator: a priobe. However, proponents of the prion hypothesis will then need to establish a consensus on the taxonomic status of the putative infective agent.

\section{Conclusion}

The principles described in this account take note of methods currently used in a variety of disciplines to assemble a robust argument for causality. While the terms used and emphasis placed on specific assertions may vary from discipline to discipline, all who work in this field understand the need to unify the evidence they present in a single, cohesive argument. Whether that argument is used to develop a hypothesis or to refine an experimental process, the process described here is itself provisional and subject to further refinement in the light of future observations.

\section{Acknowledgements}

I am grateful to my colleagues, and in particular to Dr Manfred Beilharz, Professor Thomas Riley and Professor Ian Poxton for their advice during the preparation of the original manuscript.

\section{References}

Aguzzi, A. \& Heikenwalder, M. (2006). Pathogenesis of prion diseases: current status and future outlook. Nat Rev Microbiol 4, 765-775.

Alam, M., Sultana, M., Nair, G. B., Sack, R. B., Sack, D. A., Siddique, A. K., Ali, A., Huq, A. \& Colwell, R. R. (2006). Toxigenic Vibrio cholerae in the aquatic environment of Mathbaria, Bangladesh. Appl Environ Microbiol 72, 2849-2855.

Bone, R. C. (1996). Immunologic dissonance: a continuing evolution in our understanding of the systemic inflammatory response syndrome (SIRS) and the multiple organ dysfunction syndrome (MODS). Ann Intern Med 125, 680-687.

Colwell, R. W. (1999). Biocomplexity. The 99th Annual General Scientific Meeting of the American Society for Microbiology, McCormick Place, Chicago, IL.

Costerton, J. W., Montanaro, L. \& Arciola, C. R. (2005). Biofilm in implant infections: its production and regulation. Int J Artif Organs 28, 1062-1068.

Falkow, S. (1988). Molecular Koch's postulates applied to microbial pathogenicity. Rev Infect Dis 10 (Suppl. 2), S274-S276.

Gullo, A. (1999). Sepsis and organ dysfunction/failure. An overview. Minerva Anestesiol 65, 529-540.

Inglis, T. J., Garrow, S. C., Adams, C., Henderson, M. \& Mayo, M. (1998). Dry-season outbreak of melioidosis in Western Australia. Lancet 352, 1600.

Inglis, T. J., Rigby, P., Robertson, T. A., Dutton, N. S., Henderson, M. \& Chang, B. J. (2000). Interaction between Burkholderia pseudomallei and Acanthamoeba species results in coiling phagocytosis, endamebic bacterial survival, and escape. Infect Immun 68, 1681-1686.

Inglis, T. J., Golledge, C. L., Clair, A. \& Harvey, J. (2001). Case report: recovery from persistent septicemic melioidosis. Am J Trop Med Hyg 65, 76-82.

Islam, M. S., Drasar, B. S. \& Bradley, D. J. (1990). Long-term persistence of toxigenic Vibrio cholerae 01 in the mucilaginous sheath of a blue-green alga, Anabaena variabilis. J Trop Med Hyg 93, 133-139.

Koch, R. (1884). Die Aetiologie der Tuberkulose Mitt Kaiser Gesundh 2, 1-88.

Lederberg, J. (1999). Pathogens at the crowded summit. The 99th Annual General Scientific Meeting of the American Society for Microbiology, McCormick Place, Chicago, IL.

Lipp, E. K., Huq, A. \& Colwell, R. R. (2002). Effects of global climate on infectious disease: the cholera model. Clin Microbiol Rev 15, 757-770.

Mazzarello, P. (2004). The explicative power of the rules: the case of the Koch's postulates. Med Secoli 16, 293-316 in Italian

Merritt, A., Inglis, T. J., Chidlow, G. \& Harnett, G. (2006). PCR-based identification of Burkholderia pseudomallei. Rev Inst Med Trop Sao Paulo 48, 239-244.

Popper, K. R. (1963). Conjectures and Refutations. London: Routledge and Kegan Paul.

Safdar, N., Crnich, C. J. \& Maki, D. G. (2005). The pathogenesis of ventilator-associated pneumonia: its relevance to developing effective strategies for prevention. Respir Care 50, 725-739.

Salyers, A. A. \& Witt, D. D. (2005). Bacterial Pathogenesis. Washington, DC: American Society for Microbiology.

Stevenson, M. A., Wilesmith, J. W., Ryan, J. B., Morris, R. S., Lockhart, J. W., Lin, D. \& Jackson, R. (2000). Temporal aspects of the epidemic of bovine spongiform encephalopathy in Great Britain: individual animal-associated risk factors for the disease. Vet Rec 147, 349-354.

Yokota, S., Imagawa, T., Miyamae, T., Ito, S., Nakajima, S., Nezu, A. \& Mori, M. (2000). Hypothetical pathophysiology of acute encephalopathy and encephalitis related to influenza virus infection and hypothermia therapy. Pediatr Int 42, 197-203. 